\title{
El cambio curricular desde la práctica educativa: diálogo de saberes e investigación en el aula
}

\section{Curricular change from educational practice: dialogue of knowledge and research in the classroom}

\author{
Juan Carlos Escobar Baños \\ Candidato a Doctor Interdisciplinario de Educación \\ Superior, Universidad de El Salvador \\ Jcescobarbaos77@gmail.com \\ https://orcid.org/0000-0002-7450-248X
}

Recibido 18/10/2021

Aceptado 16/11/2021

Escobar Baños, J. C. (2021). El cambio curricular desde la práctica educativa: diálogo de saberes e investigación en el aula. Revista Guatemalteca de Educación Superior, 5(1), 135-148. https://doi.org/10.46954/revistages.v5i1.81

\section{Resumen}

OBJETIVO: analizar las tradiciones curriculares como referentes teóricos del docente-investigador en la dinámica de evaluar el currículum desde las prácticas educativas. MÉTODO: se utilizó el método analítico-sintético en el abordaje de las categorías pedagógicas en el estudio de las teorías del currículum. Además, se precisó de la modelación para examinar la figura del docente-investigador en los procesos de reconstrucción y toma de decisiones; lo que permitió sistematizar el modelo de evaluación como alternativa para actualizar planes de estudio. RESULTADOS: la mayor fortaleza de la figura del docenteinvestigador es la toma de decisiones en la transformación del currículum, revitalizando el debate público y científico en la 
producción de conocimiento. Su debilidad se encuentra en la práctica reiterativa de la enseñanza técnica-ritual, centrada en el saber hacer, sin asumir un papel protagónico; lo que conlleva a la negación del diálogo de saberes y la investigación en el aula. CONCLUSIÓN: cambiar el diseño curricular desde las prácticas

Palabras clave:

práctica educativa, currículum, teoría crítica, investigación, diálogo de saberes.

\section{Keywords:}

educational practice, critical theory, currículum, research, dialogue knowledge educativas es posible si se gesta en comunidades autorreflexivas del trabajo de los docentes universitarios, dada la perspectiva de reconstruir las competencias interdisciplinarias de los estudiantes en sus prácticas profesionales.

\section{Abstract}

OBJECTIVE: to analyze the curricular traditions as theoretical referents of the teacher-researcher in the dynamics of evaluating the currículum from educational practices. METHOD: the analytical-synthetic method was used in the approach of the pedagogical categories in the study of the theories of the currículum. Furthermore, modeling was required to examine the figure of the teacher-researcher in the reconstruction and decision-making processes; This made it possible systematize the evaluation model as an alternative to update study plans. RESULTS: the greatest strength of the figure of the teacherresearcher is the decision-making in the transformation of the currículum, revitalizing the public and scientific debate in the production of knowledge. Its weakness is foundo in the repetitive practice of technical-ritual teaching, centered on know-how, without assuming a leading role; which leads to the denial of the dialogue of knowledge and the research in the classroom. CONCLUSION: changing the currículum design from educational practices is possible if it takes from educational practices is possible if it takes place in self-reflective communities of the work of university teachers, given the perspective of rebuilding the interdisciplinary competencies of students in their professional practices. 


\section{Introducción}

La concepción de cambio curricular y práctica educativa está asociada a las actuales condiciones históricas en que se encuentra el mundo contemporáneo, marcado por profundos cambios socio-culturales, sobre todo, en lo económico y político, que ha demandado reajustes o reformas educativas, a fin de repensar la formación profesional y redireccionar el proceso de enseñanzaaprendizaje con el propósito de contribuir al desarrollo del país, de acuerdo con las demandas que se exigen.

Para tales demandas sociales y educativas, el autor de esta investigación considera que el diseño curricular debe ser revisado y evaluado; es decir, tal como planteó Elliot (2005), se deben realizar procesos de rendición de cuentas, porque toda práctica educativa se debiera renovar de acuerdo con el saber científico, su pertinencia social y el nivel de calidad exigible en este ámbito del nivel universitario. Es decir, la vigencia de un currículum nacional (como lo fue en el Reino Unido en 1971) estuvo determinado por la preocupación de superar el fracaso de la enseñanza como instrumento de transformación de habilidades y destrezas de los estudiantes, tanto en la dirección del proceso de enseñanza-aprendizaje como en los resultados de su formación.

En efecto, gran parte del debate público que se generó en Inglaterra (1970-1980), consistió en incorporar a los docentes en el diseño de programas de estudio, de modo que fuesen ellos quienes tomaran las decisiones justas e informadas acerca de la pertinencia de los programas de estudio. Un cambio curricular nace de la reflexión y se desarrolla cuando los docentes "están preparados para cuestionar su propia práctica" (Grundy, 1998, pág. 137); lo que constituye el cimiento de la evaluación de los planes y programas de estudio a partir del trabajo pedagógico reflexivo y de la elaboración conjunta de juicios objetivos y prudentes. 
El presente escrito es el resultado de la indagación teórica y experiencial de la tesis doctoral acerca del significado de currículum, sus modelos principales y su importancia en la formación del licenciado en ciencias de la educación de la Universidad de El Salvador, dado la relevancia de evaluar el diseño curricular actuante como premisa de la mejora sustancial de los programas de estudio y sus implicaciones en los contenidos de enseñanza, objetivos (su derivación gradual) y competencias, métodos y técnicas, recursos didácticos y tecnológicos, actividades de aprendizaje y evaluación.

\section{Contenido}

Se abordan dos referentes teóricos. En primer lugar, se pasa revisión sobre las teorías curriculares en cuanto a tradiciones educativas, destacando el significado de currículum, planes y programas de estudio y evaluación. En segundo lugar, se presenta la figura del docente investigador, desde dos concepciones derivadas de la tradición práctica (Schwab, 1969; Stenhouse, 2003), critica (Carr \& Kemmis, 1988; Kemmis, 1993; Freire, 2016) y el diálogo de saberes en la dinámica del conocimiento del Sur (Sousa, 2019).

\section{Miradas a las tradiciones curriculares: premisas del docente como investigador}

La reivindicación de la teoría curricular se hace posible con la constitución de los sistemas educativos nacionales que, en términos generales, se ajustaron a los designios de los gobernantes y al interés por formar recursos humanos de la sociedad industrial. Popkewitz (2001) asegura que la institucionalización del currículum implicó prácticas discursivas, ligadas a la regulación y el control, que muy pronto se expandieron a todas las regiones del mundo, en especial, aquellas que habían buscado incesantemente políticas de transformación curricular, tales como: Italia, Alemania, Francia, Estados Unidos, entre otros. 
La teoría de la educación, que más adelante, se convirtió en teoría curricular, evidenció dos situaciones claves: por un lado, se colocó como prescripción y regulación de la práctica educativa, en especial a finales del siglo XIX y principios del XX, cuando se intentó asociar el currículum a las fuerzas productivas y calificadas de la sociedad (Kemmis, 1993), de modo que la reproducción social quedara adherida a los valores, formas de vida y trabajo de los patrones económicos, culturales y políticos del estado moderno. Por el otro, se definieron programas de estudio de carácter universal en el sentido de homologar la formación y garantizar la unificación de los valores socio-educativos, liberales y humanos del orden de la filosofía pragmática.

Se evidencia, desde esta óptica una primera tendencia curricular: el saber técnico. Se politiza, desde el ángulo de la sociedad industrial, las prácticas discursivas, que focalizaban la organización del currículum en torno a su concreción en cursos de enseñanza que se expandían en las escuelas y universidades, a fin de colocar en primer plano "El conocimiento como tecnología disciplinaria" (Popkewitz, 2001, pág. 115). Esto significó una apertura al saber hacer, por el nivel de importancia que se le concedió a la planificación didáctica que cada curso debía conllevar, así como el control de los conocimientos y valores que los estudiantes tienen que aprender, en correspondencia con las finalidades de este tipo de sociedad.

Se trata de la tradición curricular técnica que supone la adhesión del aprendizaje a la programación de la enseñanza (Skinner, 1970) y a la tendencia de que la educación puede mejorar siguiendo patrones o prescripciones establecidas en escenarios distintos. Lo que conlleva a determinar que la perspectiva técnica solo puede asegurar una formación profesional de calidad siempre y cuando los objetivos sean formulados con la mayor claridad posible y que el producto [que debiera ser el aprendizaje] sea evaluado en términos de medida, con las características básicas de indicadores que garanticen la división social del trabajo: "Cuanto más claramente puedan especificarse los objetivos que guían la acción docente, más previsible será el resultado" (Grundy, 1998, pág. 54). 
Los conceptos de habilidades y destrezas son cruciales en la tradición técnica, dada la importancia que se le otorga a los objetivos específicos y la evaluación de los conocimientos. Distintos autores (Stenhouse, 1998; Popkewitz, 2001)) aseguran que el currículum, desde esta perspectiva, consiste en un producto que regula la práctica educativa, una tendencia prescriptiva ligada al cambio de conducta, controlada mediante la evaluación; o lo que es equivalente a educar el razonamiento técnico. Para ello, es indispensable entrenar a los docentes en el dominio de métodos de enseñanza sobre la base de esas destrezas que deben aprender para ejecutar el currículum.

Básicamente, la concepción de currículum como entrenamiento de habilidades para el logro de objetivos operativos se acentúa en el posicionamiento de Ralph Tyler mediante su obra «Basic Principles of Curriculum and Instruction» publicada en 1949. Aquí se describe la necesidad de generalizar los estándares mínimos para una formación de calidad, asegurando, en un sentido, la integración de los objetivos nacionales con las necesidades de los estudiantes, siempre que concuerde con la nueva tecnología de una sociedad pragmática como Estados Unidos, orientada a impartir instrucción (Carr \& Kemmis, 1988). El investigador educativo, en esta concepción, no era el docente, sino un experto que suministraba instrumentos para evaluar el currículum, bajo la tutela de ciertos indicadores estandarizados.

En 1969, Schwab profundizaba en un pensamiento nuevo. Se trataba de la perspectiva curricular práctica, que pretendía soslayar la educación de la ciencia aplicada y ubicar como alternativa el protagonismo del docente en la toma de decisiones, ya que la argumentación de Tyler consistía en que los docentes debían ocuparse exclusivamente de la tecnologización de la enseñanza, sin llegar a la argumentación curricular (Kemmis, 1993); en otras palabras, la posición de Schwab (1969) generó el surgimiento de una segunda tradición curricular, concebida como saber práctico, dando la oportunidad a los docentes a reflexionar sobre el currículum ejecutado. 
Es un saber práctico, derivado de la tradición del «juicio práctico», que consiste en una forma de interpretación hermenéutica, vinculada especialmente a la comprensión de la acción y a los significados presentes en la toma de decisiones (Grundy, 1998). El campo de estudio de esta tradición es el reconocimiento y el respeto a la razón humana como emprendedora de la toma de decisiones y la responsable de la acción, dando validez a la reflexión en situación (deliberación) como una forma de justicia que se hace posible en las relaciones de los perfiles profesionales y la concreción de la práctica educativa, en las relaciones del individuo y sociedad, como problema central del currículum.

La transformación de la práctica educativa desafía el conjunto de reglas curriculares como formas de organización del conocimiento prescrito (Popkewitz, 2001). La conversión del docente en investigador tiene doble vía de concreción: por medio de redes y equipos docentes, someten a un juicio práctico la efectividad de la enseñanza y el aprendizaje para mejorar o perfeccionar las prácticas educativas. La segunda vía de concreción está en el debate público, que no solo es producto de los juicios de las autoridades pedagógicas o consultores, sino de la participación de los colegas-docentes y estudiantes, que están implicados en las realidades especificas en los espacios áulicos.

El saber práctico sugerido por Schwab fue concretado por Stenhouse cuando propone el protagonismo del docente en la construcción consciente y activa del cambio curricular. En el Humanities Currículum Project (Reino Unido, 1970), Stenhouse (1998) prioriza las experiencias educativas gestadas en el proceso de enseñanza-aprendizaje como relevantes para ser investigadas por el docente, ya que toda prueba que se da, producto del análisis y recomendaciones externas, carecen de fundamento y de proyección pedagógica para cambiar realidades.

Stenhouse (1998) precisó de una teoría práctica basada en la investigación, a fin de que los docentes revisaran los diseños curriculares y pudiesen formular los juicios críticos a partir de sus experiencias educativas contextuales. En la conferencia denominada «La difusión de la investigación-acción», Stenhouse, especifica lo siguiente: 
Al informar de la investigación, espero persuadirles de que revisen de manera crítica sus propias experiencias para comprobar después la investigación frente a su evaluación crítica de esas experiencias. No trato de defender que la investigación deba despreciar su juicio: debe complementarlo y enriquecerlo... al considerar cualquier descubrimiento de la investigación en estos términos, tendrán que plantearse a sí mismo dos cuestiones: primera, ¿es verdadero en general?, y segunda, ¿es verdadero en mi caso? (Grundy, 1998, pág. 105)

Es una investigación para analizar lo individual y lo general, tomar como punto de partida la situación real de la problemática, para profundizar en el seno de las prácticas educativas, a la cual Freire (2019) llamó a una exigencia para que el docente reconozca desde el punto de vista político, que su compromiso sea a favor de los pueblos oprimidos. En consecuencia, de esta tradición práctica, emerge la tendencia crítica del currículum, como una referencia complementaria al posicionamiento de Stenhouse en cuanto a responsabilizar a los docentes a ser los constructores del currículum. Se trata del saber crítico-emancipador, con una visión dialéctica que integra la praxis en la construcción y reconstrucción de los diseños curricular, tomando de base el eje vertebrador su condición política por el cambio social.

El diálogo libre y de saberes es una expresión viva que mantiene la tradición crítica con la realidad. De acuerdo con los estudios sobre sociología de la educación, currículum y reproducción realizados por Stephen Kemmis en 1988 (Universidad de Deakin), determinó que los investigadores debían "comprender las interpretaciones teóricas en base para el análisis de las decisiones y prácticas distorsionadas por razones sistémicas, así como sugerir el tipo de acción social y educacional que permitirían eliminar esas distorsiones" (Carr \& Kemmis, 1988, pág. 49).

Todo ello, exige que los docentes se ilustren y tomen conciencia sobre aquellos contenidos y prácticas, a veces difusas, que generan condiciones de injusticia, que impiden darse cuenta sobre cómo funcionan los mecanismos sociales y políticas que limitan la conducta adecuada de la educación. En ese camino 
dialéctico, la investigación-acción constituye una actividad fundamental que enriquece el conocimiento de los docentes para tomar decisiones sobre la dinámica del diseño curricular y práctica educativa. De este modo,

Investigación-acción es un gran templo que incorpora una serie de enfoques, así como de debates internos. En general su foco es la práctica profesional de los maestros, que reflexionan sobre su propia actuación con el objetivo de aclarar sus preocupaciones y mejorar su dominio de ellas. (Turner, 2015, pág. 59)

El currículum, en esta tradición, se somete a un proceso evaluativo para generar conocimientos y métodos para enseñar; la revisión de programas de estudio son el resultado de la docencia en acción, con una visión dialéctica, que dinamiza el diálogo de saberes (Sousa, 2019), para enriquecer la formación profesional con nuevos contenidos de enseñar y aprender, que en el trasfondo, sean claves en la disposición para actuar justa y correctamente. Se trata de un currículum abierto/flexible, que permite la interconexión con la realidad, enriqueciéndose constantemente para su revisión periódica y las respuestas que debe dar a esa realidad, que constituye su objeto de estudio.

\section{Docentes investigadores: un análisis desde las tradiciones prácticas y críticas}

La figura «El profesor como investigador» en su acepción «prolongada» fue utilizada por Stenhouse en los proyectos Humanities Currículum Project (1975) y A Study in the Dissemination of Action Research (1979) para la exploración del conocimiento a través de la práctica, de modo que los docentes se apropien de la investigación como un desafío para cambiar el mundo de la vida en las aulas. En vez de tradición práctica, Stenhouse (1998) propone el «modelo de proceso» para referirse a la reflexión como parte de la actividad docente en la reconstrucción de la práctica educativa, cuya dinámica se encuentra en la autoconciencia y en la problematización. 
Grundy (1998) esboza un enfoque de la ciencia social interpretativo a través de un saber que reconcilia el interés práctico con el crítico, referenciando posiciones racionales para que los sujetos sean capaces de emanciparse de las ideas falsas o de las estructuras que son determinantes para la reproducción social; es decir, a la vez, que se intenta interpretar el mundo (tradición práctica), la ciencias de la educación son emancipadoras (tradición crítica), que revelan cómo el poder y el currículum se asocian coercitivamente en la dinámica de la ideología dominante.

Más allá de cualquier alegato de los pedagogos y científicos sociales, la interpretación (Schwab, 1969; Stenhouse, 1998; Turner, 2015) y la emancipación (Carr \& Kemmis, 1988; Freire, 2019) no se conforman con iluminar a los investigores educativos sobre la comprensión de las relaciones sociales y los planes didácticos a ejecutar, sino a "crear las condiciones mediante las que las relaciones sociales distorsionadas existentes pueden ser transformadas en acción organizada, cooperativa, una lucha política compartida, donde las personas traten de superar la irracionalidad y injusticia que desvirtúan sus vidas" (Kemmis, 1993, pág. 88).

Sí para Stenhouse el modelo de proceso es adoptar el método comprehensivo en los diseños curriculares, para Kemmis la ciencia social busca estudiar a fondo la ideología, con una postura problematizadora acerca del conocimiento, generando en la docencia, la posibilidad de actuar políticamente en un escenario viable de investigación. Pero la propuesta de Stenhouse (1998) abrió los espacios de debate sobre el modelo de proceso del currículum, cuyo camino se logra por medio del docente investigador prolongado. Para ello, señala las siguientes características:

El compromiso para el cuestionamiento sistemático de la propia enseñanza como base para el desarrollo;

El compromiso y las destrezas para estudiar la propia enseñanza;

El interés para cuestionar y probar la teoría en la práctica mediante el uso de esas destrezas.

(Stenhouse, 1988, pág. 144) 
Stenhouse (1998b) afirmaba que el docente investigador utiliza la investigación para el desarrollo del currículum; por lo tanto, el lugar de los profesores para indagar, descubrir y reflexionar es la práctica educativa, que localiza el diseño curricular para visualizarlo como una tentativa de ser refutada o mejorada por los docentes y estudiantes en interacción, mientras la investigación vaya generando la información necesaria para la toma de decisiones. En ese transcurrir de indagación, el docente cuestiona la teoría en la práctica, adquiriendo el compromiso de estar sometiendo al escrutinio crítico el diseño curricular en el escenario específico de su propia forma de enseñanza.

No se trata de una actividad extra de los docentes, como quien proporciona una carga más a su trabajo; es un acto que dignifica a las ciencias pedagógicas y le da poder al docente para controlar los conocimientos mediante la investigación-acción. Un control que facilita evaluar su práctica $y$, al mismo tiempo, informar sobre los cambios generados que deben incorporarse en el diseño del currículum. De esta forma, la investigación curricular rompe con la tendencia positivista-técnica del currículum, hace emerger una nueva figura (sin que esto afecte la sobre carga académica del docente), que debe ser entendida a través de la propuesta de Carr y Kemmis (1988), denominada: «Comunidades autorreflexivas».

Como dice Zeichner (2010), la investigación-acción es un proceso colectivo de producción de conocimiento, llevado a cabo por los docentes que deciden deliberar y tomar decisiones, que aseguren el cambio curricular desde la práctica educativa, rompiendo con el determinismo cognitivo y la hegemonía técnica-científica que, como tal, sigue imperando en las decisiones gubernamentales y de las autoridades universitarias que a menudo rechazan el crecimiento de los equipos docentes en cuanto investigadores. No cabe duda, que una propuesta, desde su seno, resultará determinante en la formación profesional, así como el aporte de los educadores que, con su voz, quieren generar marcos de referencias para interpretar y mejorar sus prácticas. 


\section{Conclusión}

La finalización de este contenido, que de una u otra forma no pretende seleccionar categorías sobre la evaluación curricular, tiene como intención sugerir la incorporación del docente como investigador en las prácticas educativas, de modo que sirva como alternativa en la toma de decisiones para la reconstrucción social de la formación del licenciado en ciencias de la educación. Sin pretender, en absoluto, sacar aquí todas las conclusiones de esta ínfima parte de la investigación doctoral, al menos se denotarán algunas que se impone:

- Integrar las tradiciones curriculares (técnica, práctica y crítica) en el cambio curricular denotará una visión holística del fortalecimiento académico del licenciado en ciencias de la educación; un proceso que conlleva crítica y protagonismo científico-pedagógico del docente en la dinámica del diálogo de saberes y el conocimiento desde el Sur, como referencias para trabajar por una mayor justicia social.

- El cambio curricular es posible sí la investigación-acción se asume como comunidad autorreflexiva, un cuerpo colegiado de docentes que reflexionan sobre su propia práctica de enseñar defienda la justicia social y ofrezcan una educación de alta calidad para incidir en la reconstrucción social y pedagógica de las competencias interdisciplinarias de los estudiantes en sus prácticas profesionales.

\section{Referencias}

Carr, W., \& Kemmis, S. (1988). Teoría crítica de la enseñanza. La investigación-acción en la formación del profesorado. Martínez Roca. Obtenido de https://asdrubaljaimes10. files.wordpress.com/2019/07/kemmis-s-y-w-carr-teoriacritica-de-la-ensenanza-1986-copia.pdf

Elliot, J. (2005). Investigación-acción en educación (Quinta ed.). Morata. Obtenido de https://www.terras.edu.ar/ biblioteca/37/37ELLIOT-Jhon-Cap-1-y-5.pdf 
Freire, P. (2019). El maestro sin recetas. Siglo XXI. Obtenido de https://www.perlego.com/book/1918789/el-maestrosin-recetas-pdf (Original work published 2019)

Grundy, S. (1998). Producto o práxis del currículum (Tercera ed.). Morata. Obtenido de https://www.academia. edu/26016411/LECTURA_OBLIGATORIA_UF_10_ Producto_o_praxis_del_currículum_Grundy_S

Kemmis, S. (1993). El currículum: más allá de la teoría de la reproducción (Cuarta ed.). Morata. Obtenido de file://C:/Users/Juan\%20Carlos/Downloads/343440365Currículum-Mas-Alla-de-La-Teoria-de-La-ReproduccionKemmis-pdf.pdf

Popkewitz, T. (2001). La producción de la razón y el poder: historia del currículum y tradiciones intelectuales. En M. Auguirre, Rostros históricos de la educación. Miradas, estilos, recuerdos. (págs. 97-139). Fondo de Cultura Económica. Obtenido de https://www.researchgate. net/publication/285250627_La_produccion_de_ razon_y_poder_Historia_del_currículum_y_tradiciones_ intelectuales

Schwab, J. (1969). The Practical: A Languaje for Currículum (Trad. Lo práctico: Un lenguaje para el currículum). The School Review(78), 1-23. Obtenido de https://www.jstor. org/stable/1084049

Skinner, B. (1970). Conferencia de revisión: La Tecnología de la enseñanza). Official B. F. Skinner Foundation Reprint Series / paperback edition. Obtenido de http://www. jstor.org/stable/75554

Sousa, B. D. (2019). Educación para otro mundo posible. CLACSO. Obtenido de http://biblioteca.clacso.edu. ar/clacso/se/20190712045634/Educacion_para_otro_ mundo_posible_Boaventura.pdf

Stenhouse, L. (1998). Investigación como base de la enseñanza (Cuarta ed.). Morata. Obtenido de https://es.scribd. com/document/336127678/Stenhouse-La-InvestigacionComo-Base-de-La-Ensenanza-pdf 
Stenhouse, L. (2003). Investigación y desarrollo del currículum.

Morata. Obtenido de https://es.scribd.com/

document/394097233/Investigacion-y-Desarrollo-Del-

Currículum-Stenhouse-2003

Turner, D. (2015). Teoría de la educación. Siglo XXI. Obtenido de https://www.perlego.com/book/1871230/teora-dela-educacin-pdf (Original work published 2016)

Zeichner, K. (2010). La formación del profesorado y la lucha por la justicia social. Morata. Obtenido de https:// www.perlego.com/book/1923783/la-formacin-delprofesorado-y-la-lucha-por-la-justicia-social-pdf (Original work published 2012)

\section{Sobre el autor}

Juan Carlos Escobar Baños, graduado de Licenciado en Ciencias de la Educación y Maestro en Profesionalización de la Docencia, de la Universidad de El Salvador. Catedrático universitario desde 1989, desarrollando asignaturas orientadas a las ciencias de la educación. Autor de diversas obras: Nociones generales de teoría de la enseñanza. Autor: Nociones generales de teoría de la enseñanza (2000); Currículum: Más allá de la teoría de la enseñanza (2007). Coautor del Diccionario Enciclopédico de Ciencias de la Educación, con Joao Picardo y Rolando Balmore Pacheco.

\section{Conflicto de intereses}

Declara no tener ningún conflicto de intereses.

\section{Declaración de consentimiento informado}

El estudio se realizó con fines educativos, respetando el Código de ética y buenas prácticas editoriales de publicación.

\section{Derechos de uso}

